prolapsed iris it has proved of singular benefit, and in one very marked case last week at St. Mary's Hospital, it saved from excision a large portion of iris which must have been removed. A little boy had received a clean cut, which had opened the cornea freely near the sclerotical junction, and without inflicting other injury. The lad was placed under chloroform in the theatre, and the eye carefully examined, which, as be was so excessively restless, could not otherwise be satisfactorily accomplished. The wound proved to be so shaped as to leave loose a small triangular tlap of cornea. Fully a fourth of the iris prolapsed. A small square of Calabar bean paper had an hour previously been introiuced under the eyelids, and all the pupil was firmly contracted except the prolapsed part; the traction was not sufficient to draw this in. It was returned by a probe, and a further drop of the glycerine extract of $J$. Bell and $\mathrm{Co}$ (one drop equal to four grains of the extract) was introduced. The lid was closed, and a pad strapped over it. All went well next morning when the eye was examined. but as the effect of the bean wore off the prolapse again occurred, as the small corneal flap had not united. Fresh instillation of the Calabar bean was made, and the eye rebandaged. By continuing this treatment, reduction has been fully established.

Mr. Hart has also been carrying out a somewhat lengthened series of observations on patients submitted to the ophthalmoscope, of which the object has been to ascertain the smallest quantity of atropine which will effect the dilatation of the pupil. The reason for wishing to ascertain this, he states, is twofold. The atropine paper prepared by Mr. Squire for Mr. Streatfeild he had found by far too strong for ordinary ophthal moscopic purposes, and far stronger, indeed, than is generally required. Thus it not only rapidly dilates the pupil, but paralyzes completely the accommodation of the eye; and these very inconvenient conditions, with the consequent disturbance of vision, often last as long as five, six, or more days after the use of this paper. Moreover, the Calabar bean paper hitherto prepared has not proved sufficiently strong to counterbalance the degree of dilatation. The square of an eighth of an inch of this paper contains $\frac{1}{40}$ of a grain of sulphate of atropia. Mr. Hart has been using paper so prepared that one square contains as little as $\frac{1}{20000}$ or $\frac{1}{100000}$ of a grain, and he finds that dilatation is very effectually procured with the latter. The dilatation is more rapid when the eye is kept in the dark immediately after the introduction of the paper, but it takes place quickly enough when the eye is exposed to the light, and lasts nearly twenty. four hours. The accommodation is very slightly affected when this paper is used, and recontraction may then be effectually obtained with the Calabar bean paper. Mr. Hart is continuing the experiments, which will enable him to fix the lowest available strength of the atropine paper or solution, and the relative strength of the Calakar bean paper or solution. Meantime it is evident that the problem of concurrent dilatation and recontraction is solved, and that surgeons wishing to dilate the pupil will find an advantage in employing the extremely dilute strength above mentioned. The papers used in the above ex. periments were supplied by Messrs. Savory and Moore.

One of the difficulties of making Calabar bean paper of considerable strength arises from the fact that at present the alco. holic extract is the most condensed preparation yet obtained, containing its active principle; and this is of course far more bulky than the alkaloid atropine. Dr. Christison has for some time, Mr. Hart observed, been endeavouring to extract from the Calabar bean an alkaloid or other active principle. 'This, however, has not yet been isolated, and it seems difficult to ascertain even what is the nature of this active principle. The bean does not yield it to acid solutions; and hence Dr. Squire has suggested that it is probably not an alkaloid, or some salt of it would be soluble in water, which appears not to be the case, as aqueous solutions of the Calabar bean are inert. Hence it seems probable that it is some body of the nature of santonine or piperine, which belong to the class of neutral or slightly acid bodies; or it may even be a resin. But it is hardly probable that the bean will yield an alkaloid; it yields a beautiful gum.

\section{WESTMINSTER HOSPITAL.}

INTESTINAL OBSTRUCTION PRODUCED BY A CONGENTTAL DIVERTICULUM PROCEEDING FROM THE ILEUM, STRANGULATING A COIL OF INTESTINE.

(Under the care of Dr. RaDCLIFFE.)

Fon the notes of the following case we are indebted to Mr. Gandy, the house-physician :-
Charles $\mathrm{C}$ - aged eight years, was admitted on the 19 th of February, 1862. His father stated that six days previous, after partaking of supper, he retired to bed, and in the night awoke complaining of cramps in the inside and pain in the hypogastric region; he shrieked and cried at intervals, and became very feverish and thirsty. The next day he is reported to have druak an immense quantity of cold water, which however returned as fast as he took it; the vomiting was constant up to the time of his admission, the ejecta being clear and limpid, with an offensive odour; the urine had been free, and he had eaten nothing since the time of the attack. Mustard and bran poultices had been applied to the abdomen, and he had had some powders and medicine to take; but there had been no action of the bowels frum the $13 \mathrm{th}$ to the $19 \mathrm{th}$. At the time of admission his countenance was anxious and pale; the eyes seemed deep-set, and the pupils were dilated. He dozed off every few minutes. The eyelids remaining half open, and the eyes being turned up, he presented a very cadaveric appearance. The tongue was somewhat dry, pasty, and covered with brown fur ; the abdomen was very much distended, with prominent. veins on the surface. Decubitus dorsal, with his knees drawn up. He breathed almost exclusively with the upper part of the chest. Since this morning he has been vomiting a yellowish brown fluid, with a disagreeable, sour, feculent smell; the skin rather cold; pulse 117 , wiry ; respiration 28 ; urine free. An enema was administered, and returned directly, without bringing away any feculent matter.

Feb. 20th. - The enemas were continued every four hours during the night, but without producing any effect. He passed a restless night, and seemed in great pain. He vomited stercoraceous matter about five A.M., and twice since. His feet and hands are cold; the abdomen swollen, but not very tense; pain is referred to no particular spot on pressure; eyes sunken tongue brown and somewhat dry; surface of the body cold and pale; pulse 90 , very feeble-scarcely perceptible at wrist ; lips dry; teeth covered with sordes. He is very thirsty; takes nothing but cold water. No tumour of any kind in the ordinany situation of hernia. The vomiting continued the whole day the symptoms were unrelieved, no freal matter returning with the injections. At a quarter to six P.M. he became delirious, and rapidly died.

Autopsy, twenty hours after death.-Body emaciated; eyes sunken and hollow; rigor mortis present; abdomen distended, and of a dark, dusky, bluish hue. On making a section through the abdominal parietes, the coils of the small intestine, which were very much distended, at once protruded themselves ; their vessels were congested, but there was no appearance of any inflammatory action. In the right lumbar and iliac regions, the distended intestine was found suddenly to terminate, being firmly constricted by a diverticulum, proceeding from the ileum; from this point the small intestine was collapsed, and of a dark, unhealthy aspect as far as its entrance into the cæcum, at which spot the unhealthy appearance ceased, and the cæcum and colon, though empty, were quite natural in appearance. On further examination, the constriction was. found to be in the ileum, about twelve inches prior to its ter mination. In the cæcum, a coil of it was firmly held by the diverticulum. On cutting it open, two ulcerated surfaces were observable in the ileum, but no perforation had taken place. The intestine above the seat of stricture contained much yellowish-brown, feculent matter, of a sourish, offensive odour, similar to some found in the stomach, and identical with that which had been vomited during life. The mesenteric glands were enlarged; the gall-bladder was distended, and full of black bile; the liver was healthy, its abdominal surface being discoloured black. The other viscera were healthy.

\section{ST. GEORGE'S HOSPITAL.}

ULCERATION OF THE VERMIFORM APPENDIX, PROCEEDTNG TO PERFORATION AND FATAL PERITONITIS.

(Under the care of Dr. BARCLAY.)

JAMES $\mathrm{N} \longrightarrow$, aged thirty-one, was admitted on the 27 th of May last. According to the patient's account, he was in excellent health three days before admission, but on that day he had taken some aperient medicine because he thought his bowels were a little sluggish. The next day, after dinner, he was attacked with most acute pain in the abdomen, which he attributed to the beer he had just drunk. The pain, however, did not yield to any of the common remedies for colic; and on the 27th of May he was brought to the hospital. 
When first seen he was in great pain; the legs were drawn up to the chest, he was afraid to speak above a whisper, and the bory gnivered with agony. The abdomen was not very hard; pulse 136 ; skin hot. On the 28 th of May he was thought a trifle better; the abclomen was a derree softer, the pulse quiet r and he had soine sleep. When first anmitted, he was ordered two grains of calomel and one grain of opium every six hours. Turpentine fomentations were now applied to the belly. He passed two quiet nights; but on the morning of the $30 \mathrm{th}$, severe pain seized him across the lower part of the abdomen. An opiate injection was at once given, but without effect. At two P.M. he was cold and clammy, and monning from pain. The pulse could not be counted. He passed a night of great pros tration, and died at seven A.M. on the 3lst.

Autopsy, thirty hours after death. - The abdomen was tym panitic; the body rather green and discoloured. The ribs were united to the sternum by ossification of the cartilages. The heart and valves were healthy. There were a few adhesions in the right pleura. The lower lobes of both lungs were congested and somewhat friable. The affected parts did not sink in water. Both surfaces of the peritoneum were coated with a rongh layer of puriform lymph. This was everywhere bathed with creamy pus. These products were distributed over the entire cavity; but they were most abundant about the right iliac fossa. When the soft adhesions between the cæcum and neighbouring bowels were broken down, a small body of the size of a cherry-stone, and much like it in appearance, was found lying in the peritoneal cavity, close to the vermiform appendix. This body proved to be a freal concretion. It was ovoid in shape. On section, crescentic laminæ of a somewhat brittle material were seen. The appendix cæci was laid open by nlceration, so that a probe could be passed from the large in testine into the cavity of the peritoneum. The mucons mem. brane near to the cæeum, for about a quarter of an inch, was natural ; elsewhere it was perforated in several places by ulcers, and the end was apparently destroyed. The liver was coated with lymph, except at its extreme left. The other viscera were normal in themselves, but were all more or less coated with soft lymph.

\section{MIDDLESEX HOSPITAL.}

OASES ILLUSTRATING THE IDENTITY OF TINEA TONSURANS AND HERPES CIRCINATUS.

(Under the care of Dr. MURCHISON.)

Agnes $\mathrm{M}-$ - aged nine, and James $\mathrm{M} \longrightarrow$-, aged eleven, presented themselves as out-patients at the above hospital in September, 1863. The boy had a patch of tinea tonsurans on the scalp as large as a crown-piece. His sister, who was supposed to have caught the disease from him, had two similar, but smaller, patches of tinea tonstirans on the scalp, and also a patch of well-marked herpes circinatus, the size of a florin, on the forehead. The patches of tinea tonsurans exhibited, under a high magnifying power, abundance of characteristic examples of the minute fungus called Tricophyton. The same fungus, but in less quantity, was found in the scales from the patch of herpes circinatus. The disease had existed in the boy for about a month. In the girl the disease on the scalp di ant commence until about ten days later, and on the forehead not until a week before she came under observation. The disease in both patients was cured by a few applications of a strong solution of bichloride of mercury (one scruple to the ounce).

\section{LONDON FEVER HOSPITAL.}

CASES ILEUSTRATING THE IDENTITY OF TINEA TONSURANS AND HERPES CIRCINATUS.

(Under the care of Dr. MURCHISON.)

MARY Axne E-, aged nine, and Emily E-, aged seven, were admitted on August $27 \mathrm{th}, 1863$, suffering from scarlet fever. Both patients had several large patches of tinea tonsurans on the scalp. Their brother, aged only six months, admitted at the same time with scarlet fever, had several patches of herpes circinatus, the size of a shilling, scattered over the neek. The mother, who was an intelligent woman, stated that her two eldest children had caught the disease on their head from a family of children, who had spots on the skin like those on the infant, and she supposed that they must have given the disease to their brother. The Tricophyton was dis covered in abundance in the scalp disease of both the girls, and in smaller quantity in the cutaneous eruption of the boy. It is worth mentioning that in all these instances the disease disappeared from the skin, without any local application, during convalescence from the scarlet fever. The two girls remained in hospital, on account of different complications, upwards of seven weeks, and by the end of that time a vigorous crop of hair had appeared un the patches of scalp formerly diseased. The attack of scarlet fever seemed to unfit the skin as a soil for the growth of the Tricophyton.

\section{atleoiral Soxieties.}

\section{MEDICAL SOCIETY OF LONDON.}

ABSTRACT OF DR. ROUTH'S LETTSOMIAN LECTURES, ON SOME POINTS CONNECTED WITH THE PATHOLOGY, DIFEERENTIAI DOAGNOSIS, AND TREATMENT OF FIBROUS TUMOURS OF THE UTERUS.

\section{LECTURE II. - On some points connected with the Differential} Diagnosis of Fibroid Tumours of the Uterus.

Dr. Routh did not propose taking all examples of disease likely to be mistaken for fibrous disease of the uterus. He would first speak of certain symptoms, and test their value; and then make an application of them, especially in regard to pregnancy and ovarian disease. But before doing so he would call attention, first, to the position in which a patient should be examined. No one alone would suffice: on the back, on all-fours, on each side, and in the erect position, were all necessary positions occasionally. He showed how cases of short pediculated ovarian disease might in this way be diagnosed from uterine disease. The sound also penetrated only in some positions. Adhesions were likewise made out by changes in position. They conll also be made out, if in the true pelvis, by the "kinometer," an instrument devised by him, and which he showed to the Society; by its introduction per vaginam the downward respiratory movement could be measured in its action upon the uterus, or in its absence make out its fixity or impaction. Hence it determined pelvic cellulitis or hæmatocele. In addicion, it showed the pulsatory movement, which indicated the degree of vascularity present. The author then dwelt on the necessity of using two sounds at a time, to determine in case of fibroids the true direction and position of the growth in its relations to the uterine cavity, the bladder, and the rectum. One was placed in the bladder and one in utero, or one in the rectum and the other in utero, and their distance determined. An instrument devised by him, called the "pelvimeter," was next exhibited. This consisted of two sounds, so adapted that they could be introduced separately, and yet locked at different lengths and angles; at the bottom of the instrument was appended a register. On ex. traction, and when again set at the points previously noted, the exact measure of the growth could be determined. If the bladder was not as long as the uterus, or if from circumstances the posterior part of the tumour could not be reached by the rectum, the size of the tumour could still be made out by it. The thickness of the abdominal walls could be determined by one blade in the bladder and the other over the abdominal parietes. Then, if the distance between the latter point and the uterine cavity were taken, this, minus the thickness of the abdominal wall, was equal to the breadth of the anterior ute. rine wall. The same plan, slightly modifier, per rectum, would determine the thickness of the posterior wall. Where the tumour was high up, you could thus of ten determine the thickness of the nterine walls when you could not reach either by the finger, and so in operations avoid catting through the peritonenm.

Another most important point, the author observed, was to become fully acquainted with all details of the previous history, as often the touch and palpation were insufficient.

Proceeding then to test the value of particular symptoms, he referred-

I. To the physical hardness, roundness, and solidity of fibroids; and he showed that even a thin fluid, $\dot{a}$ fortiori a viscid one, enclosed tightly in an inelastic bag, might convey to the feel the idea of a solid. This was proved by an experiment, conducter on the table, with a bullock's bladder, hardened in spirit for a week, and in to which water was forcibly injected. In a very short time it lost all feel of fluctuation; covered by a cloth, it appeared purely solid. He then instanced 